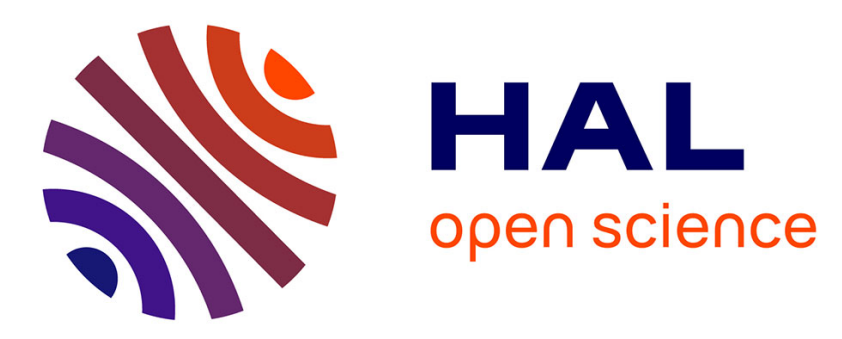

\title{
Binary partition tree-based local spectral unmixing
} Lucas Drumetz, Miguel Angel Veganzones, Ruben Marrero, Guillaume

Tochon, Mauro Dalla Mura, Antonio Plaza, Jocelyn Chanussot

\section{To cite this version:}

Lucas Drumetz, Miguel Angel Veganzones, Ruben Marrero, Guillaume Tochon, Mauro Dalla Mura, et al.. Binary partition tree-based local spectral unmixing. WHISPERS 2014 - 6th Workshop on Hyperspectral Image and Signal Processing: Evolution in Remote Sensing, Jun 2014, Lausanne, Switzerland. pp.n/c. hal-01010427

\section{HAL Id: hal-01010427 https://hal.science/hal-01010427}

Submitted on 19 Jun 2014

HAL is a multi-disciplinary open access archive for the deposit and dissemination of scientific research documents, whether they are published or not. The documents may come from teaching and research institutions in France or abroad, or from public or private research centers.
L'archive ouverte pluridisciplinaire HAL, est destinée au dépôt et à la diffusion de documents scientifiques de niveau recherche, publiés ou non, émanant des établissements d'enseignement et de recherche français ou étrangers, des laboratoires publics ou privés. 


\title{
BINARY PARTITION TREE-BASED LOCAL SPECTRAL UNMIXING
}

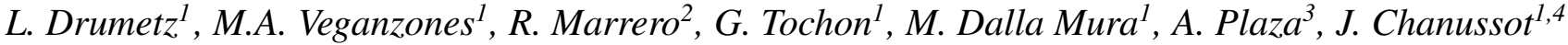 \\ ${ }^{1}$ GIPSA-lab, Grenoble-INP, Saint Martin d'Hères, France \\ ${ }^{2} \mathrm{IPAG}$, Grenoble, France \\ ${ }^{3}$ Hyperspectral Computing Laboratory, University of Extremadura (UEX), Cáceres, Spain \\ ${ }^{4}$ Faculty of Electrical and Computer Engineering, University of Iceland, Reykjavik, Iceland
}

\begin{abstract}
The linear mixing model (LMM) is a widely used methodology for the spectral unmixing (SU) of hyperspectral data. In this model, hyperspectral data is formed as a linear combination of spectral signatures corresponding to macroscopically pure materials (endmembers), weighted by their fractional abundances. Some of the drawbacks of the LMM are the presence of multiple mixtures and the spectral variability of the endmembers due to illumination and atmospheric effects. These issues appear as variations of the spectral conditions of the image along its spatial domain. However, these effects are not so severe locally and could be at least mitigated by working in smaller regions of the image. The proposed local SU works over a partition of the image, performing the spectral unmixing locally in each region of the partition. In this work, we first introduce the general local SU methodology, then we propose an implementation of the local SU based on a binary partition tree representation of the hyperspectral image and finally we give an experimental validation of the approach using real data.
\end{abstract}

Index Terms - Hypespectral imaging, local spectral unmixing, spectral bundles, binary partition trees.

\section{INTRODUCTION}

The spectral unmixing (SU) decomposes a hyperspectral image into a set of spectral signatures corresponding to macroscopically pure materials, named endmembers, and a set of cover proportions comprised in a fractional abundance matrix. The linear mixing model (LMM), is the most widely used formulation of the spectral unmixing problem, where the endmembers and fractional abundances linearly combine to form the image. A common way to perform the spectral unmixing is to determine the endmembers in the scene by picking them from a spectral library or by inducing them directly from the image, and estimating their corresponding abundances in a second step, for all pixels in the image.

The capability of the LMM to accurately obtain the set of endmembers and the abundances depends to a large extent on the presence of multiple mixtures [1] and the spectral variability of the endmembers due mainly to illumination and atmospheric effects [2]. Some algorithms such as the Multiple Endmember Spectral Mixture Analysis (MESMA) [3] address the former issue, but they are computationally expensive and make use of spectral libraries. The latter has been addressed by either statistical methods that treat the endmembers as multivariate statistical variables [4], or by the use of

L. Drumetz was supported by ERC AdG-2012-320684 CHESS. Miguel A. Veganzones was supported by ERC AdG-2013-320594 DECODA and by the Delegation Generale de l'Armement (DGA) under contract PDE2012600079. spectral bundles [5] (i.e., sets of spectral signatures that implicitly define the spectral variability of an endmember). The high dimensionality and between-bands correlation of hyperspectral data make the use of statistical methods very challenging. The use of spectral bundles requires the imposition of hard sparse constraints in order to obtain the abundances.

Here we propose a novel approach that faces these issues by performing the spectral unmixing locally, that is, in subsets of the image spatial domain, and then combines the results of the multiple local spectral unmixing processes to obtain the spectral and spatial decomposition of the image. The motivation behind this approach is two-fold: i) the illumination variabilities and atmospheric effects are less severe locally than when the whole image is considered, and ii) we assume that the existence of multiple mixtures is related to their spatial location and then, it is likely that in a local context we are dealing with a single mixture. By independently unmixing each of the regions in a partition of the image, we avoid the difficulties due to effects that appear globally, when the whole image is unmixed. In order to find a meaningful partition of the image to perform the local spectral unmixing (LSU), we propose the use of a binary partition tree (BPT) representation of the image and a pruning strategy directed by the estimated quality of the unmixing process. We show that the use of a LSU approach obtains better reconstruction errors than the global approach over a real data set.

The remainder of the paper is as follows: in Secs. 2 and 3 we overview the linear spectral unmixing and the binary partition tree representation of hyperspectral images, respectively. In Sec. 4 we define the local spectral unmixing approach, and in Sec. 5 the proposed BPT-based local spectral unmixing. In Sec. 6 and 7 we present the experimental methodology and give the experimental results, respectively. Finally, we make some conclusion remarks in Sec. 8.

\section{SPECTRAL UNMIXING}

Let $\mathbf{E}=\left[\mathbf{e}_{1}, \ldots, \mathbf{e}_{m}\right]$ be the pure endmember signatures in the hyperspectral image, where each $\mathbf{e}_{i} \in \mathbb{R}^{q}$ is a $q$-dimensional vector. Then, the LMM states that an hyperspectral signature $\mathbf{x}_{j}$ at $j$-th pixel is defined by the expression:

$$
\mathbf{x}_{j}=\sum_{i=1}^{m} \mathbf{e}_{i} \phi_{j i}+\mathbf{n}_{j},
$$

where $\mathbf{n}_{j}$ denotes an independent additive noise component, and $\phi_{j}=\left[\phi_{j 1}, \ldots, \phi_{j m}\right]$ is the $m$-dimensional vector of fractional per-pixel abundances. The fractional abundances are constrained to match physical properties by the abundance non-negative (ANC), $\phi_{i j} \geq 0, \forall i, \forall j$, and the abundance sum-to-one (ASC), $\sum_{i=1}^{m} \phi_{i j}=$ $1, \forall j$, constraints. Without the noise, the hyperspectral pixels in 
the LMM (1) lie inside a simplex whose vertexes are the endmembers. This geometrical property has led to the development of multiple endmember induction algorithms (EIA) that estimate the endmembers from the hyperspectral data set $[6,7]$. Once the set of endmembers, $\hat{\mathbf{E}}$, has been estimated from the data or selected from a spectral library, their fractional abundances can be obtained by an optimization process:

$$
\hat{\boldsymbol{\phi}}_{j}=\arg \min _{\boldsymbol{\phi}_{j} \geq \mathbf{0}}\left\|\mathbf{r}_{j}-\hat{\mathbf{E}} \boldsymbol{\phi}_{j}\right\|_{2}^{2}+\tau\left\|\boldsymbol{\phi}_{j}\right\|_{1}
$$

s.t. $\sum_{i=1}^{m} \phi_{i j}=1$, where $\|\cdot\|_{p}$ denotes the $p$-norm. The second term in (2) is a sparsity term that depends on the value of the sparsity factor, $\tau$. When $\tau=0$, Eq. (2) reduces to the classic constrained least squares form.

\section{BINARY PARTITION TREES}

In the BPT representation, the leaf nodes correspond to an initial partition of the image, which can be the individual pixels, or a coarser segmentation map [8]. From this initial partition, an iterative bottom-up region merging algorithm is applied until only one region remains. This last region represents the whole image and corresponds to the root node. All the nodes between the leaves and the root result of the merging of two adjacent children regions. An example of BPT is displayed in Fig. 1. If the initial partition contains $n$ leaf nodes, the final BPT contains $2 n-1$ nodes.

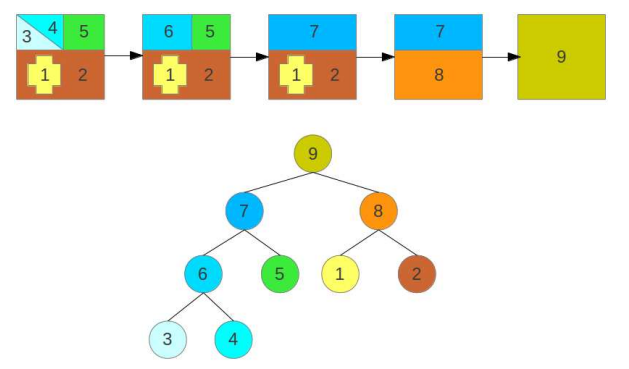

Fig. 1. Construction of the Binary Partition Tree (BPT).

Two notions are of prime importance when defining a BPT: i) the region model $\mathcal{M}_{k}$ which specifies how a region $\mathbf{X}_{k}$ is modelled, and ii) the merging criterion $\mathcal{O}\left(\mathcal{M}_{\alpha}, \mathcal{M}_{\beta}\right)$, which is a distance measure between the region models of any two regions $\mathbf{X}_{\alpha}$ and $\mathbf{X}_{\beta}$. Each merging iteration involves the search of the two adjacent regions which achieve the lowest pair-wise similarity among all the pairs of adjacent regions in the current segmentation map. Those two regions are consequently merged. Given a hyperspectral region $\mathbf{X}_{k}$, with $N_{k}$ hyperspectral samples $\mathbf{x}_{k}, k \in 1 \ldots N_{k}$, the first-order parametric model $\mathcal{M}_{k}$ [9] is defined by the sample mean vector of the hyperspectral samples $\hat{\boldsymbol{\mu}}_{k}$ :

$$
\mathcal{M}_{k}: \hat{\boldsymbol{\mu}}_{k}=\frac{1}{N_{k}} \sum_{k=1}^{N_{k}} \mathbf{x}_{k}
$$

Using the first-order parametric model (3), a merging criterion is defined as the spectral angle distance, $d_{\mathrm{SAD}}$, between the sample mean vectors of any two adjacent regions [10]:

$$
\mathcal{O}\left(\mathcal{M}_{\alpha}, \mathcal{M}_{\beta}\right): d_{\mathrm{SAD}}\left(\hat{\boldsymbol{\mu}}_{\alpha}, \hat{\boldsymbol{\mu}}_{\beta}\right)
$$

where $d_{\mathrm{SAD}}(\mathbf{a}, \mathbf{b})=\arccos \left(\frac{\mathbf{a}^{T} \mathbf{b}}{\|\mathbf{a}\|\|\mathbf{b}\|}\right)$.

The building of a BPT may suffer from small and meaningless regions resulting in a spatially unbalanced tree. To overcome this limitation, a priority term is included in the merging criterion that forces those regions smaller than a given percentage of the average region size to be merged first [11]. Often, the BPT is pruned to achieve a segmentation where the nodes of the pruned tree represent an optimal partition for some kind of applications.

\section{LOCAL SPECTRAL UNMIXING}

Let $\mathcal{P}$ denote a partition of a hyperspectral image $\mathbf{X}$ :

$$
\mathcal{P}(\mathbf{X})=\left\{\mathbf{X}_{k}\right\}_{k=1}^{K},
$$

s.t. $\mathbf{X}_{i} \cap \mathbf{X}_{j}=\emptyset, \forall i, j=1, \ldots, K, i \neq j$; and $\bigcup_{k=1}^{K} \mathbf{X}_{k}=$ $\mathbf{X}$. The LSU methodology consists of estimating, independently for each region, $\mathbf{X}_{k}$, the endmembers, $\hat{\mathbf{E}}_{k}$, and the fractional abundances, $\hat{\mathbf{\Phi}}_{k}=\left\{\hat{\phi}_{k j}\right\}$, using the pixels indexed by the region, $\mathbf{x}_{j} \in$ $\mathbf{X}_{k}$. The locally estimated abundances, $\hat{\boldsymbol{\Phi}}_{k}$, form a partition of the image abundance matrix, $\mathcal{P}(\hat{\boldsymbol{\Phi}})=\left\{\hat{\boldsymbol{\Phi}}_{k}\right\}_{k=1}^{K}$.

The sets of endmembers identified for each region can be grouped together in a single set, $\mathcal{E}=\bigcup_{k=1}^{K} \hat{\mathbf{E}}_{k}$, that contains a sampling of the spectral variability within the whole image. Then, the endmembers in $\mathcal{E}$ can be clustered, i.e. by means of a spectral angle distance-based K-Means, to obtain a set of spectral bundles representing the spectral variability of the materials in the image:

$$
\mathbf{B}_{i}=\left\{\tilde{\mathbf{e}}_{i l}\right\}_{l=1}^{L_{i}},
$$

s.t. $\mathbf{B}_{i} \bigcap \mathbf{B}_{j}=\emptyset, \forall i, j=1, \ldots, m$; and $\bigcup_{i=1}^{m} \mathbf{B}_{i}=\mathcal{E}$, where $\tilde{\mathbf{e}}_{i}$ denotes a spectral variation of an endmember $\mathbf{e}_{i}$ due to illumination or atmospheric effects, and $L_{i}$ denotes the total number of spectral variations for the $i$-th endmember. Thus, the LSU works as a methodology to locally estimate the spectral bundles representation of the endmembers in a hyperspectral image. Note that even if the spectral meaning of the LSU is explained as spectral bundles, this is a posterior step to the unmixing process and then, it differs from the common use of spectral bundles for spectral unmixing.

\section{BPT-BASED LOCAL SPECTRAL UNMIXING}

Let $\Omega=\{\mathcal{P}(\mathbf{X})\}$ be the set of all possible partitions of the hyperspectral image $\mathbf{X}$ given by a BPT representation of the image. In the following, we propose two pruning criteria based on the LSU of the partitions in $\Omega$. The pruning criteria serve to obtain a partition of the image that is optimal in terms of its local spectral unmixing. Each proposed pruning criterion searches for the partition minimizing either the average or the maximum of a reconstruction error quality measure, $\epsilon(\mathbf{x}, \hat{\mathbf{x}})$, where $\hat{\mathbf{x}}=\hat{\mathbf{E}} \hat{\boldsymbol{\phi}}$ is the reconstruction of the hyperspectral vector $\mathbf{x}$ by the estimated endmembers and corresponding fractional abundances. Any reconstruction error quality measure could be used, i.e. the root mean squared error or the spectral angle distance. The pruning criterion based on the average of the error is defined as:

$$
\mathcal{P}_{\text {avg }}^{\star}(\mathbf{X})=\arg \min _{\mathcal{P}(\mathbf{X}) \in \Omega} \frac{1}{N} \sum_{\mathbf{X}_{k} \in \mathcal{P}(\mathbf{X})} \sum_{\mathbf{x} \in \mathbf{X}_{k}} \epsilon(\mathbf{x}, \hat{\mathbf{x}}),
$$

where $N$ is the number of pixels in the image. Similarly, the partition minimizing the overall maximum reconstruction error is defined as

$$
\mathcal{P}_{\max }^{\star}(\mathbf{X})=\arg \min _{\mathcal{P}(\mathbf{X}) \in \Omega} \max _{\mathbf{x}} \epsilon(\mathbf{x}, \hat{\mathbf{x}}), \forall \mathbf{x} \in \mathcal{P}(\mathbf{X}) .
$$




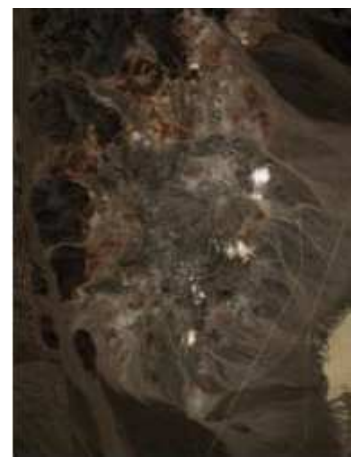

Fig. 2. A false color representation of the Cuprite scene.

It is sometimes interesting to constrain the set of valid partitions, $\Omega$, to those containing regions above a minimum spatial size. In these cases, the set $\Omega$ of valid partitions in (7) and (8) is replaced by the subset of size-constrained valid partitions, $\Omega_{c}$ :

$$
\Omega_{c}=\{\mathcal{P}(\mathbf{X}) \in \Omega, \text { s.t. } \forall \mathcal{R} \in \mathcal{P}(\mathbf{X}),|\mathcal{R}| \geq c\},
$$

where $|\mathcal{R}|$ denotes the cardinality (number of pixels) of region $\mathcal{R}$ and $c \geq 0$ is a threshold on the region size.

\section{EXPERIMENTAL METHODOLOGY}

We compare the proposed LSU approach to the conventional one using the whole image, and hereafter, named as global spectral unmixing (GSU), to induce the endmembers and estimate the corresponding abundances. We test the two approaches using the Cuprite dataset [12] (see Fig. 2). The scene was taken by the NASA's AVIRIS sensor and covers the Cuprite mining district in western Nevada, USA.

We define the experimental unmixing process as the application of the Vertex Component Analysis (VCA) [13] to induce the endmembers from a given set of pixels. The VCA is run 10 times per region in order to avoid issues due to its stochastic nature, retaining the set of endmembers with maximum simplex volume. Then, the unmixing process estimates the abundances corresponding to the induced endmembers and the given set of pixels by solving the optimization problem in (2) with sparsity, $\tau=10^{-3}$, and without sparsity, $\tau=0$.

For the case of the GSU, the unmixing process is run over the whole data set. For the LSU, we have implemented the proposed BPT-based LSU (Sec. 5), where the BPT representation is built using the first order parametric model (3) and the merging criterion defined in (4). Then, the unmixing process is independently run over each node of the BPT representation in order to find the optimal partition given by the two pruning strategies (7) and (8) using as the reconstruction error, $\epsilon(\mathbf{x}, \hat{\mathbf{x}})$, the root mean squared error (RMSE) and the spectral angle distance (SAD):

$$
\begin{aligned}
\epsilon_{\mathrm{RMSE}}(\mathbf{x}, \hat{\mathbf{x}}) & =\sqrt{\frac{1}{q} \sum_{t=1}^{q}\left(x^{(t)}-\hat{x}^{(t)}\right)^{2}} \\
\epsilon_{\mathrm{SAD}}(\mathbf{x}, \hat{\mathbf{x}}) & =\arccos \left(\frac{\mathbf{x}^{T} \hat{\mathbf{x}}}{\|\mathbf{x}\|\|\hat{\mathbf{x}}\|}\right) .
\end{aligned}
$$

The pruning criteria were constrained by a region minimum size parameter (9) set in the range $c \in[100: 1000]$ with step size of 5 .

\section{RESULTS}

Figs. 3 and 4 show the average reconstruction errors (RMSE and SAD, respectively) obtained by the LSU and GSU approaches. The horizontal axis shows the number of regions in the partition obtained by the pruning criterion according to the value of the region size constraint $c$. As the value of $c$ increases, the obtained partitions contain a lower number of regions, but eventually a range of close $c$ values yields to the same optimal partition. The value obtained for the global approach is shown as a constant value for a better visual comparison, but there is only one region as the global approach is applied to the whole image. The BPT-based LSU approaches outperform the global ones in all cases except for the maximum RMSE pruning criterion with sparse-constrained abundance estimation, where the average SAD values are worse. The pruning criteria based on the minimization of the mean reconstruction error (7) obtain in most cases the best average RMSE and SAD results independently of the reconstruction error selected for the optimization, with partitions containing few regions (less than 20) in the case of the abundances obtained without sparsity.

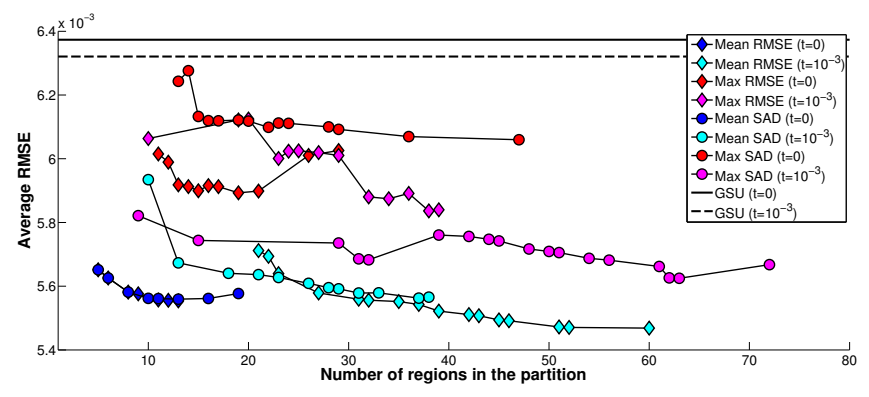

Fig. 3. Average RMSE reconstruction errors of the LSU with the different pruning strategies and the GSU. The $t$ parameter denotes the sparsity factor set for the abundance estimation.

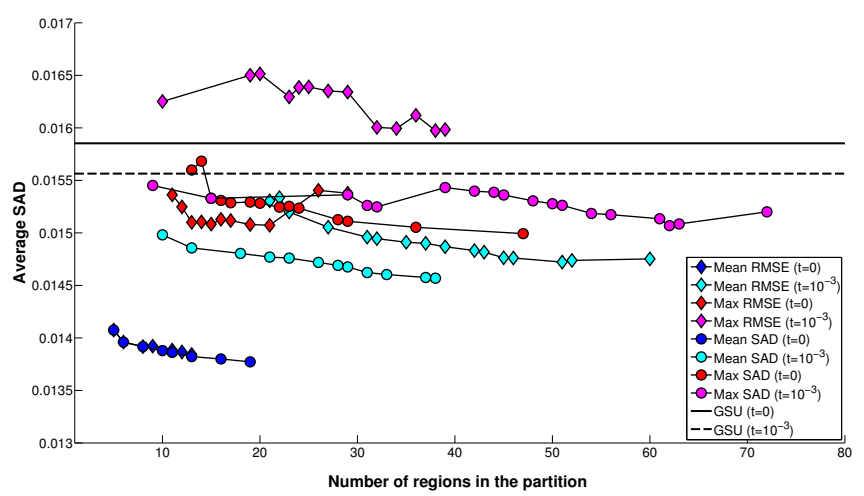

Fig. 4. Average SAD reconstruction errors of the LSU with the different pruning strategies and the GSU. The $t$ parameter denotes the sparsity factor set for the abundance estimation.

Fig. 5 shows the segmentation maps obtained by the mean RMSE pruning criterion when the size constraint is set to $c=100$, that is, the reconstruction errors corresponding to the rightmost 
points of the LSU plots in Figs 3 and 4 (dark and light blue diamonds). When no sparsity is enforced, most of the regions in the obtained partition are covering the variability located in the rightmost part of the image. However, when sparsity is enforced, there are also a set of regions covering the leftmost part of the image. In both cases the largest region covers the most homogeneous area of the image. This suggests that even if the reconstruction errors are slightly better for the non-sparse pruning, the use of sparsity could be more reasonable in terms of spectral variability representation and abundance estimation.

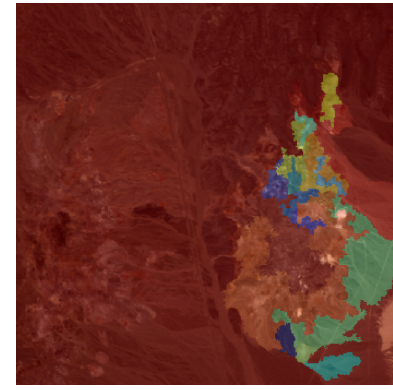

(a)

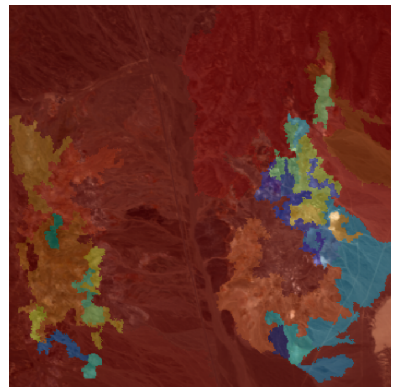

(b)
Fig. 5. Partition obtained from the BPT representation by the mean RMSE pruning criterion with region size constraint set to $c=200$ : a) without sparsity $(t=0)$ and, b) with sparsity $\left(t=10^{-3}\right)$.

The computational times required to build the BPT representations and populate them with the unmixing information using a 64bits 4 core Intel Xeon(R) CPU at $2.80 \mathrm{GHz}$ is about 28 mins. The computational time is mainly due to the unmixing process (which is run ten times for each region), since the computational cost of the BPT construction and the posterior pruning is almost negligible. However, this approach is well suited for parallelization, and further efforts will be done to reduce the computational burden by taking advantage of the hierarchical structure to populate the tree with the unmixing information.

\section{CONCLUSIONS}

We have introduced a novel general approach based on the local spectral unmixing of hyperspectral images in order to overcome the limitations of the classical global spectral unmixing when there are several mixtures of materials or spectral variability due to illumination and atmospheric effects. We have also proposed a BPT-based implementation of local spectral unmixing. Results show that the proposed approach yield better reconstruction errors, suggesting that it is able to cope with these issues. We concluded that this a promising methodology that requires of further work to completely understand the nuances of the proposed BPT-based local spectral unmixing. We will focus on the use of BPT representations built over an unmixing-based model and merging criterion, on the evaluation of the proposed local spectral unmixing in more depth, with real and synthetic data, in terms of reconstruction quality but also in terms of abundances estimation. Also, we need to explore the unmixing information contained through the spatial scales, and make an effort to reduce the computational burden.

\section{REFERENCES}

[1] A. Zare, P. Gader, O. Bchir, and H. Frigui, "Piecewise convex multiple-model endmember detection and spectral unmixing,"
Geoscience and Remote Sensing, IEEE Transactions on, vol. 51, no. 5, pp. 2853-2862, May 2013.

[2] A. Zare and K.C. Ho, "Endmember variability in hyperspectral analysis: Addressing spectral variability during spectral unmixing," IEEE Signal Processing Magazine, vol. 31, no. 1, pp. 95-104, Jan 2014.

[3] D.A. Roberts, M. Gardner, R. Church, S. Ustin, G. Scheer, and R.O. Green, "Mapping chaparral in the santa monica mountains using multiple endmember spectral mixture models," $R e$ mote Sensing of Environment, vol. 65, no. 3, pp. 267 - 279, 1998.

[4] N. Dobigeon, S. Moussaoui, M. Coulon, J-Y Tourneret, and A.O. Hero, "Joint bayesian endmember extraction and linear unmixing for hyperspectral imagery," IEEE Transactions on Signal Processing, vol. 57, no. 11, pp. 4355-4368, Nov 2009.

[5] B. Somers, M. Zortea, A. Plaza, and G.P. Asner, "Automated extraction of image-based endmember bundles for improved spectral unmixing," IEEE Journal of Selected Topics in Applied Earth Observations and Remote Sensing, vol. 5, no. 2, pp. 396408, April 2012.

[6] J.M. Bioucas-Dias, A. Plaza, N. Dobigeon, M. Parente, Qian $\mathrm{Du}, \mathrm{P}$. Gader, and J. Chanussot, "Hyperspectral unmixing overview: Geometrical, statistical, and sparse regression-based approaches," IEEE Journal of Selected Topics in Applied Earth Observations and Remote Sensing, vol. 5, no. 2, pp. 354-379, April 2012.

[7] W.-K. Ma, J.M. Bioucas-Dias, Tsung-Han Chan, N. Gillis, P. Gader, A.J. Plaza, A. Ambikapathi, and Chong-Yung Chi, "A signal processing perspective on hyperspectral unmixing: Insights from remote sensing," IEEE Signal Processing Magazine, vol. 31, no. 1, pp. 67-81, Jan 2014.

[8] Philippe Salembier and Luis Garrido, "Binary partition tree as an efficient representation for image processing, segmentation, and information retrieval," IEEE Transactions on Image Processing, vol. 9, no. 4, pp. 561-576, 2000.

[9] S. Valero, P. Salembier, and J. Chanussot, "Hyperspectral image representation and processing with binary partition trees," IEEE Transactions on Image Processing, vol. 22, no. 4, pp. 1430-1443, 2013.

[10] M.A. Veganzones, G. Tochon, M. Dalla Mura, A. Plaza, and J. Chanussot, "Hyperspectral image segmentation using a new spectral mixture-based binary partition tree representation," in 2013 IEEE International Conference on Image Processing (ICIP), 2013.

[11] F. Calderero and F. Marques, "Region merging techniques using information theory statistical measures," IEEE Transactions on Image Processing, vol. 19, no. 6, pp. 1567-1586, June 2010.

[12] R.O. Green, M.L. Eastwooda, C.M. Sarturea, T.G. Chriena, M. Aronssona, B.J. Chippendalea, J.A. Fausta, B.E. Pavria, C.J. Chovita, M. Solisa, M.R. Olaha, and O. Williamsa, "Imaging spectroscopy and the airborne visible/infrared imaging spectrometer (aviris)," Remote Sensing of Environment, vol. 65, no. 3, pp. $227-248,1998$.

[13] J.M.P. Nascimento and J.M. Bioucas-Dias, "Vertex component analysis: a fast algorithm to unmix hyperspectral data," IEEE Transactions on Geoscience and Remote Sensing, vol. 43, no. 4, pp. 898 - 910, april 2005. 